\title{
Das Istanbul-Protokoll und die Dokumentation von Folter
}

\author{
Holger Furtmayr/Andreas Frewer
}

\section{Inhaltsübersicht}

\section{Einleitung}

II. Die Problematik der Folter und ihrer Dokumentation

III. Zur Entstehung des Istanbul-Protokolls

IV. Struktur und Gliederung des Textes

V. Inhalte und Prinzipien des IstanbulProtokolls

VI. Umsetzung und Implementierung VII. Schlußüberlegungen

\section{Einleitung}

Kaum etwas vermag einen Menschen körperlich und seelisch so zu zerstören, wie die Folter. Neben den Schmerz und die Todesangst tritt die sichere Gewißheit, daß die erlittenen Qualen keine unvermeidlichen und zufälligen Naturkatastrophen sind, sondern von Mitmenschen gezielt zugefügt werden. Sie stellen das Opfer bloß und isolieren es - auch von seiner Familie und seinem nächsten Umfeld. Folter betrifft deshalb nie nur den Einzelnen: Sie zerstört das für jede Gemeinschaft unerläßliche Vertrauen in den Anderen und wirkt damit auf einen Kreis, der weit über den Gefolterten hinausgeht. Genau 60

\footnotetext{
Wir danken der Staedtler-Stiftung in Nürnberg für die Förderung des Forschungsprojekts zur Edition des Istanbul-Protokolls. Darüber hinaus möchten wir insbesondere Dr. Kerstin Krása (Erlangen), Prof. Thomas Wenzel (Wien) und Thomas Oberschmidt (Berlin) für die gute $\mathrm{Zu}$ sammenarbeit im Rahmen des Projektes danken. Der Leitung der Friedrich-AlexanderUniversität Erlangen-Nürnberg und der Medizinischen Fakultät danken wir für die Förderung des „Forum Medizin und Menschenrechte ${ }^{\prime \prime}$ an der Professur für Ethik in der Medizin des Instituts für Geschichte und Ethik der Medizin der Universität Erlangen-Nürnberg.
}

Jahre nach der Allgemeinen Erklärung der Menschenrechte ${ }^{1}$ und beinahe 25 Jahre nach der Verabschiedung des Übereinkommens gegen Folter (CAT) ${ }^{2}$ sowie zwei Jahrzehnte nach der Einsetzung des UNAusschusses gegen Folter zur Überwachung dieses Abkommens, besteht Folter weiterhin und wird gegenwärtig sogar in über 80 Ländern dieser Erde eingesetzt. ${ }^{3}$

Da die betroffenen Staaten selbst offensichtlich höchstens zögerlich willens sind, das Problem anzugehen, ist es umso wichtiger, auf eine sorgfältige Dokumentation von Fällen zu drängen, bei denen der Verdacht besteht, daß Folter stattgefunden hat. Das wichtigste Instrument hierzu ist das so genannte Istanbul-Protokoll, ${ }^{4}$ dem damit letztendlich auch eine zentrale Rolle für die Prävention von Folter zukommt. Das Istanbul-Protokoll kam anläßlich eines internationalen Symposiums der türkischen

1 Vom 10. Dezember 1948, UN-Dok. A/810, S. 71; dt. z.B. abgedruckt in: Sartorius II Nr. 19.

2 Übereinkommen gegen Folter und andere grausame, unmenschliche oder erniedrigende Behandlung oder Strafe vom 10. Dezember 1984, UNTS Bd. 1465, S. 85; BGBl. 1990 II, S. 247.

3 Amnesty International, Amnesty International Report 2008 - Zur weltweiten Lage der Menschenrechte, 2008.

4 Das Istanbul-Protokoll wird auch als "Manual on the Effective Investigation and Documentation of Torture and other Cruel, Inhuman and Degrading Punishment or Treatment" (Handbuch für die wirksame Untersuchung und Dokumentation von Folter und anderer grausamer, unmenschlicher oder erniedrigender Behandlung oder Strafe) bezeichnet. Die englische Version ist erhältlich unter: www.ohchr.org/ Documents/Publications/training8Rev1en.pdf. Bisher ist das Istanbul-Protokoll in den offiziellen Sprachen der UNO (arabisch, chinesisch, englisch, französisch, russisch, spanisch) erschienen. 
Ärztekammer 1996 unter Mitwirkung von Gerichtsmedizinern, Ärzten, Menschenrechtsbeobachtern und Rechtsanwälten zustande. ${ }^{5}$ Die grundlegende Intention des Protokolls ist es, internationale Richtlinien aufzustellen, nach denen eine sorgfältige Ermittlung der Faktenlage bei einem Verdacht auf Folter ermöglicht wird, so daß die gewonnenen Befunde auch in einem strafrechtlichen Verfahren als Beweismittel Bestand haben. ${ }^{6}$ Entstanden aus der täglichen Arbeit und der Notwendigkeit einer sorgfältigen Dokumentation von Foltervorfällen (siehe II, unten), verstehen sich die aufgestellten Richtlinien dabei nicht als feststehende Vorschriften, sondern vielmehr als flexible Ratschläge für die Praxis, die den jeweiligen Gegebenheiten und vorhandenen Ressourcen angepaßt werden müssen. ${ }^{7}$ Neben dem Ziel, die für Folter Verantwortlichen strafrechtlich zur Rechenschaft zu ziehen, kann die Dokumentation von Foltervorfällen mit Hilfe des Istanbul-Protokolls aber auch in anderen Zusammenhängen von Nutzen sein, beispielsweise bei der Untersuchung von Menschenrechtsverletzungen oder bei der Begutachtung von mutmaßlichen Folteropfern im Rahmen von Asylverfahren. Darüber hinaus soll das Handbuch Anhaltspunkte für den Behandlungsbedarf von Folteropfern liefern und letztendlich auch zu einer "Wiedergutmachung", sofern dies bei einem solchen Verbrechen überhaupt möglich ist, für die Opfer und deren Familien führen. Diese vielfältigen Möglichkeiten machen die Anwendung des IstanbulProtokolls nicht nur in solchen Ländern sinnvoll, in denen Folter weiterhin syste-

$5 \quad \mathrm{Zu}$ den Einzelheiten siehe unten, Punkt III.

6 "The broad purpose of the investigation is to establish the facts relating to alleged incidents of torture, with a view to identifying those responsible for the incidents and facilitating their prosecution [...]", Istanbul-Protokoll, S. 17, § 77.

7 "The guidelines contained in this manual are not presented as a fixed protocol. Rather, they represent minimum standards based on the principles and should be used taking into account available resources.", Istanbul-Protokoll, S. 2 . matisch verübt wird, sondern auch in denjenigen, wo Folteropfer in erster Linie als Flüchtlinge auftreten. ${ }^{8}$ Aus diesem Grund wird das an der Professur für Ethik in der Medizin der Friedrich-Alexander-Universität Erlangen-Nürnberg bestehende „Forum Medizin und Menschenrechte" in Kürze eine deutsche Fassung des IstanbulProtokoll herausgeben. ${ }^{9}$ Damit ist die Intention verbunden, dieses Handbuch für die wirksame Untersuchung und Dokumentation von Folter und anderer grausamer, unmenschlicher oder erniedrigender Behandlung oder Strafe auch im deutschsprachigen Raum einem größeren Kreis von praktizierenden Menschenrechtlern und Menschenrechtlerinnen, sowie vor allem Ärzten und Ärztinnen und Juristen und Juristinnen bekannt zu machen.

\section{Die Problematik der Folter und ihrer Dokumentation}

Im aktuellen Jahresbericht 2008 hat Amnesty International in 81 Staaten Fälle von Folter oder anderer entwürdigender und unmenschlicher Behandlung dokumentiert. ${ }^{10}$ Insbesondere angesichts des in das gleiche Jahr fallenden 60. Jahrestags der Allgemeinen Erklärung der Menschenrechte offenbart diese Zahl die frappierende Diskrepanz zwischen dem Bekenntnis der Staaten gegen jede Form von Folter und deren tat-

$8 \quad \mathrm{Zu}$ den verschiedenen Verwendungsmöglichkeiten der Daten, die bei einer medizinischrechtlichen Dokumentation von Folter nach den Standards des Istanbul-Protokoll gewonnen wurden, insbesondere in Behandlungszentren für Folteropfer, siehe: Lene Mandel/Lise Worm, Documentation of torture victims. Implementation of medico-legal protocols, in: Torture 2007, Vol. 17/1, S. 18-26.

9 Andreas Frewer/Holger Furtmayr/Kerstin Krása/ Thomas Wenzel (Hrsg.), Istanbul-Protokoll. Handbuch für die wirksame Untersuchung und Dokumentation von Folter und anderer grausamer, unmenschlicher oder erniedrigender Behandlung oder Strafe, 2008.

10 Amnesty International (Fn. 3); einen Überblick über die wichtigsten Zahlen und Fakten aus diesem Bericht gibt es unter www.amnesty.de/ files/JB08ZahlenFakten08.pdf (12. August 2008). 
sächlicher Anwendung. Dabei sind die rechtlich weitgehend unverbindlichen Bestimmungen der Allgemeinen Erklärung der Menschenrechte mittlerweile längst durch eine Reihe von Verträgen zu bindendem Recht für die einzelnen Vertragsstaaten geworden. $\mathrm{Zu}$ nennen sind hier an erster Stelle der Internationale Pakt über bürgerliche und politische Rechte (Zivilpakt) von 197611 und das Übereinkommen gegen Folter und andere grausame, unmenschliche oder erniedrigende Behandlung oder Strafe (CAT) von 1984 mit seinem Fakultativprotokoll,12 das 2006 in Kraft trat. Beide Dokumente enthalten ein kategorisches Verbot der Anwendung von Folter, das unter absolut allen äußeren Umständen Geltung beansprucht, so daß es keinerlei Rechtfertigung für die Anwendung von Folter oder anderer entwürdigender Behandlung geben kann. ${ }^{13}$ Und beide wurden von einer überwältigenden

11 International Covenant on Civil and Political Rights vom 16. Dezember 1966, UNTS Bd. 999, S. 171; BGBl. 1973 II, S. 1534.

12 Fakultativprotokoll zum Übereinkommen gegen Folter und andere grausame, unmenschliche oder erniedrigende Behandlung oder Strafe vom 18. Dezember 2002, UN-Dok. A/RES/57/199, Annex. Das Fakultativprotokoll verpflichtet Vertragsstaaten, nationale Kommissionen einzurichten, die der Überwachung der UN-Anti-Folter-Konvention dienen. Diese werden unterstützt von einem internationalen Unterausschuß. Die Kommissionen haben das Recht, alle Orte zu besuchen, an denen Menschen gegen ihren Willen festgehalten werden, also neben Gefängnissen auch psychiatrische Einrichtungen oder den Transitbereich von Flughäfen. Darüber hinaus müssen die Regierungen der Kommission alle benötigten Informationen, wie beispielsweise die Gründe für eine Festnahme, zur Verfügung stellen. Siehe hierzu ausführlich: Claudia Mahler, Das Fakultativprotokoll der Konvention gegen Folter und andere grausame, unmenschliche oder erniedrigende Behandlung oder Strafe (CAT-OP), in: MRM 2003, S. 183-186.

13 Das Übereinkommen gegen Folter legt beispielsweise in Art. 2 (2) fest: „Außergewöhnliche Umstände gleich welcher Art, sei es Krieg oder Kriegsgefahr, innenpolitische Instabilität oder ein sonstiger öffentlicher Notstand, dürfen nicht als Rechtfertigung für Folter geltend gemacht werden".
Mehrheit aller Staaten unterzeichnet bzw. anerkannt. Daß es trotz dieser Eindeutigkeit, sowohl was das Bekenntnis gegen jede Art der Folter als auch die rechtliche Verbindlichkeit genannter Verträge anbelangt, dennoch in so weitem Ausmaß zu einem Bruch mit geltendem Recht kommen kann, liegt teilweise in der Struktur der Vereinten Nationen begründet.

Als intergouvernementaler Zusammenschluß von unabhängigen Staaten waren alle Mitglieder von Anfang an darauf bedacht, die volle Souveränität in ihren jeweiligen Hoheitsgebieten $\mathrm{zu}$ behalten. Trotz einer seit den 1990er Jahren feststellbaren Tendenz, über eine weite Auslegung dessen, was eine „Bedrohung für den internationalen Frieden" darstellt, bei großflächigen und schwerwiegenden Verstößen gegen die Menschenrechte auch zu militärischen Maßnahmen zu greifen und beispielsweise bei Bürgerkriegskonflikten in Drittstaaten einzugreifen, ${ }^{14}$ ist das Souveränitätsprinzip weitestgehend in Kraft. Dies bedeutet, daß die einzelnen Staaten selbst für die Einhaltung und Durchsetzung der oben genannten Verträge verantwortlich sind. Es existiert kein übergeordneter Sanktionsmechanismus, um einen Verstoß gegen das hierin festgeschriebene Recht zu ahnden. Da es sich bei dem Verbot der Folter um ein Abwehrrecht des einzelnen gegenüber dem Staat handelt, und Folterhandlungen gemäß Art. 1 Abs. 1 CAT gerade dadurch definiert sind, daß sie

von einem Angehörigen des öffentlichen Dienstes oder einer anderen in amtlicher Eigenschaft handelnden Person, auf deren Veranlassung oder mit deren ausdrücklichem oder stillschweigendem Einverständnis verursacht werden,

entsteht so ein Dilemma: Es muß mit hoher Wahrscheinlichkeit damit gerechnet werden, daß diejenigen staatlichen Organe, die eigentlich dafür Sorge tragen müßten, das Folterverbot $\mathrm{zu}$ überwachen und rechtlich durchzusetzen, denjenigen Organen nahestehen, die gerade für die Folter verant-

14 Sven Bernd Gareis/Johannes Varwick, Die Vereinten Nationen, 2003, S. 223ff. 
wortlich sind. Ein Staat, der zur Folter greift, wird wenig Interesse an einer Aufklärung haben und daran, die verantwortlichen Täter vor Gericht zu stellen. ${ }^{15}$

Trotz dieser Kritik muß natürlich auch gesehen werden, daß es erst die Vereinten Nationen ermöglicht haben, der Folter praktisch weltweit öffentlich eine klare Absage zu erteilen. So gibt es, auch wenn die von Amnesty International jüngst vorgelegten Zahlen wenig erfreulich sind, dennoch positive Entwicklungen: Da Folter inzwischen weltweit geächtet wird und weil mittlerweile in fast allen Staaten Folterhandlungen rechtlich verboten sind, selbst dort, wo sie de facto stattfinden, kann es sich praktisch kein Staat erlauben, sich öffentlich $\mathrm{zu}$ einer Anwendung von Folter $\mathrm{zu}$ bekennen. Selbst die USA, als einzige verbliebene militärische Weltmacht und "Hauptbeitragszahler" der Vereinten Nationen, die im Zuge ihres so genannten „Kampfes gegen den Terror" wieder zu einzelnen Formen von Folter greifen, ${ }^{16}$ bemühen sich darum, die Bedeutung des Begriffs „Folter“ derart neu zu definieren, daß die von ihnen verwendeten Methoden der „fortgeschrittenen Vernehmungstechniken“ oder der „,verschärften Vernehmung" (enhanced interrogation techniques, harsh interrogation) nicht als solche zählen. ${ }^{17}$ Wo Folter heute stattfindet, muß sie

15 In dem Verfahren Ismail Alan ./. Schweiz hat der Ausschuß gegen Folter festgestellt, daß die Ratifizierung der UN-Anti-Folter-Konvention „noch nichts über die tatsächliche Situation in dem betreffenden Staat aussage." Hierzu Norman Weiß, Auswertung der Rechtsprechung des Ausschusses gegen Folter (CAT), in: MRM 1997, S. 15-22.

16 Siehe hierzu auch den Beitrag von Margarita Georgas/Gunda Meyer/Udo Moewes, Menschenrechte in Filmen - Wie werden Menschenrechte in Filmen dargestellt? Drei Beispiele, in diesem Heft.

17 Zu den Bemühungen, die das US-amerikanische Justizministerium hierfür unternahm, siehe beispielsweise: Hans Leyendecker, Die Lügen des Weißen Hauses in: Psychosozial 2005, Nr. 100, Heft II, S. 15. In diesem Zusammenhang zur Unterscheidung von Folter und anderer grausamer, unmenschlicher oder erniedrigender Behandlung: Manfred Nowak/Elizabeth McArthur, verheimlicht und vertuscht werden. Nichts bringt demnach Folterer mehr in Bedrängnis, als wenn sie an das Licht der Öffentlichkeit gezerrt, ihre Taten aufgeklärt und sorgfältig dokumentiert werden. Genau an diesem Punkt setzt das Istanbul-Protokoll an. Das Handbuch für die wirksame Untersuchung und Dokumentation von Folter und anderer grausamer, unmenschlicher oder erniedrigender Strafe oder Behandlung gibt internationale Richtlinien vor und benennt Untersuchungsmethoden und Standards, die eine genaue Dokumentation von Foltervorfällen ermöglichen, sie öffentlich machen und so zu einer Aufklärung über Folter beitragen. Die in dem IstanbulProtokoll enthaltenen Prinzipien stellen dabei zwar kein verbindliches Recht dar, sondern verstehen sich als Ratschläge und detaillierte Hinweise für die tägliche Praxis von Ärzten und Ärztinnen, Juristen und Juristinnen sowie anderen Experten und Expertinnen, die mit der Untersuchung von Foltervorfällen befaßt sind. Dennoch besitzen diese Richtlinien einen quasiverbindlichen Charakter, weil jeder Staat sich an sie halten muß, will er behaupten, daß er tatsächlich eine sorgfältige und wirksame Untersuchung von Folteranschuldigungen durchführt. ${ }^{18}$

The distinction between torture and cruel, inhuman or degrading treatment, in: Torture 2006, Vol. 16/3, S. 147-151.

18 Eventuell kann die Tatsache, daß das IstanbulProtokoll als UN-Dokument anerkannt ist (siehe III, unten) sowie die Bezeichnung als „Protokoll“ den Eindruck erwecken, es handle sich um ein rechtsverbindliches Dokument. Vgl. Jan Ole Haagensen, The role of the Istanbul-Protocol in the uphill battle for torture survivors being granted asylum in Europe and ensuring the perpetrators pay, in: Torture 2007, Vol. 17/3, S. 236-239 (S. 238).

Über die tatsächlich vorhandene rechtliche Verpflichtung, jeden Verdacht auf Folter umgehend und unparteiisch zu untersuchen und die Verantwortlichen strafrechtlich $\mathrm{zu}$ verfolgen, erhält das Handbuch unter Umständen doch eine gewisse Verbindlichkeit, da es die hierfür erforderlichen Maßnahmen benennt. Siehe hierzu: Hemme Battjes, Legal effects of the Istanbul Protocol, in: René Bruin/Marcel Reneman/Evert Bloemen, CARE FULL. Medico-legal reports and the 


\section{Zur Entstehung des Istanbul- Protokolls}

Konkreter Anlaß für die Entstehung des Istanbul-Protokolls und zugleich beispielhafter Beleg für das oben skizzierte Dilemma war der Fall Baki Erdoğan:19 Am 10. August 1993 wurde Erdoğan in seiner Heimatprovinz Aydin in der Türkei wegen des Verdachts auf eine Mitgliedschaft in der verbotenen "Revolutionären Linken“ verhaftet. Zehn Tage später lag er im Koma und wurde in ein Krankenhaus gebracht, wo er kurz darauf verstarb. Als offizielle Todesursache wurde zunächst ein Lungenödem angegeben, hervorgerufen durch einen Hungerstreik. Während Familienangehörige den Leichnam Erdoğans wuschen, konnten sie jedoch überall Spuren von Folterhandlungen erkennen. Bei der Beerdigung lenkten einige Familienmitglieder die anwesenden Polizisten ab, während andere das Leichentuch aufrissen und den Leichnam fotografierten und filmten. Auf der Grundlage dieser Hinweise erstellte die Ärztekammer von Izmir ein alternatives Gutachten, das als Todesursache ein durch multiple Traumata verursachtes akutes Lungenversagen nennt sowie Stromschläge und Folter durch Hängen. Darüber hinaus hat die Ärztekammer das Attest der offiziellen Gerichtsmedizin für ungültig er-

Istanbul Protocol in asylum procedures, 2006, S. 16-29.

19 Über die genauen Hintergründe des Falls Baki Erdoğan gibt es in den deutschsprachigen Informationsquellen teilweise widersprüchliche Angaben. Unser besonderer Dank gilt an dieser Stelle Dr. Alp Ayan von der Human Rights Foundation of Turkey (HRFT) für weiterführende Informationen und einige Klarstellungen. Siehe hierzu auch: Knut Rauchfuss, Das IstanbulProtokoll und die Folter in: Infobrief Nr. 97 des Republikanischen Anwältinnen- und Anwältevereins, abrufbar unter www.rav.de/ infobrief97/Rauchfuss.html (22. September 2008); Amnesty International, Jahresbericht 1994, Länderbericht Türkei, abrufbar unter http://aidrupal.aspdienste.de/umleitung/ 1994/deu03/002?lang=de\%26mimetype\%3dtext \%2fhtml (22. September 2008); Amnesty International, Turkey - The duty to supervise, investigate and prosecute, 1999, abrufbar unter http://asiapacific.amnesty.org/library/Index/ ENGEUR440241999?open\&of=ENG-376. klärt, weil es nicht entsprechend der Prinzipien des sogenannten MinnesotaProtokolls erstellt wurde. Dieses Protokoll, das Referenzpunkt der Ermittlung war, enthält Richtlinien für die Untersuchung von extralegalen und willkürlichen Hinrichtungen. ${ }^{20}$ In der Folge kam es zu einem Prozeß und schließlich zu einer Verurteilung der Täter, die jeweils fünfeinhalb Jahre Haft wegen fahrlässiger Tötung erhielten.

Als die türkische Ärztekammer im März 1996 in Adana ein internationales Symposium zu dem Thema "Medizin und Menschenrechte" abhielt, ergriff die Menschenrechtsstiftung der Türkei (TIHV, oder englisch: "Human Rights Foundation of Turkey" /HRFT) zusammen mit den Physicians for Human Rights (PHR) die Initiative, um nach dem Vorbild des MinnesotaProtokoll eine Sammlung von Richtlinien zu erstellen, die der Untersuchung von Foltervorfällen an noch lebenden Opfern dienen sollte. ${ }^{21}$ Während diese beiden Organisationen das Projekt koordinierten und organisierten, waren an der Entstehung des Protokolls letztendlich aber über 75 Experten beteiligt, die mehr als 40 Organisationen aus 15 Ländern vertraten. Die endgültige Version des Istanbul-Protokolls ist das Ergebnis einer dreijährigen gemeinsamen Analyse, Forschung und Arbeit am Text, ausgeführt von Gerichtsmedizinern, Ärzten, Psychologen, Menschenrechtsbeobachtern und Rechtsanwälten, unter denen sich sowohl Frauen als auch Männer befanden.

Nach seiner Fertigstellung wurde das Protokoll im August 1999 der damaligen UN-

20 "Principles on the Effective Prevention and Investigation of Extra-legal, Arbitrary and Summary Executions"; zu erhalten unter: www2.ohchr.org/english/law/executions.htm (22. September 2008).

21 Zur Entstehungsgeschichte des Protokolls siehe auch Vincent Iacopino/Öder Özkalipçi/Caroline Schlar, The Istanbul Protocol: international standards for the effective investigation and documentation of torture and ill treatment, in: The Lancet 1999; Vol. 354, S. 1117 sowie Hulyar Ucpinar/Turkan Baykal, An important step for prevention of torture, in: Torture 2006 (Vol. $16 / 3)$, S. 252-267. 
Hochkommissarin für Menschenrechte, Mary Robinson, übergeben. Der Empfehlung des UN-Sonderberichterstatters über Folter folgend, wurde das Protokoll am 4. Dezember 2000 sowohl von der Generalversammlung als auch von der Menschenrechtskommission angenommen. ${ }^{22}$ Noch in der gleichen Sitzung wurde über eine Veröffentlichung und breitere Bekanntmachung der Richtlinien zur Untersuchung und Dokumentation von Folter diskutiert. Im März 2001 erschien das IstanbulProtokoll schließlich im Rahmen der UNAusbildungsreihe in den sechs offiziellen UN-Sprachen und ist in diesen auf der Webseite des Büros des Hochkommissars für Menschenrechte der Vereinten Nationen erhältlich. ${ }^{23}$ Auch die Europäische Union und die Afrikanische Menschenrechts- und Völkerrechtskommission haben das Protokoll als effektives und geeignetes Mittel zur Aufklärung und Dokumentation von Foltervorwürfen anerkannt.

\section{Struktur und Gliederung des Textes}

Das Istanbul-Protokoll liegt mittlerweile in deutscher Übersetzung vor und gliedert sich in die auf der nächsten Seite dargestellten Abschnitte. ${ }^{24}$

22 Resolution 55/89 der Generalversammlung vom 4. Dezember 2000 und Resolution 2000/43 der Menschenrechtskommission vom 20. April 2000.

23 UN-Professional Training Series No. 8, abrufbar unter www.ohchr.org/EN/Publications Resources/Pages/TrainingEducation.aspx (12. August 2008).

24 An dieser Stelle werden nur die Hauptkapitel (I-V) und die Unterkapitel (A, B, C etc.) in der Form der ersten deutschen Gesamtübersetzung wiedergegeben. Die nächste Unterkategorie der Gliederung (jeweils 1., 2., 3. usw.) ist aus Platzgründen nicht dargestellt. Siehe detailliert in Frewer et al. (Fn. 9).

\section{Inhalte und Prinzipien des Istanbul- Protokolls}

Da das Istanbul-Protokoll als Handbuch für die Praxis entwickelt wurde, sind die darin enthaltenen Richtlinien und Bestimmungen, wie bereits dargestellt, nicht einklagbar. Dennoch bilden eine Vielzahl von internationalen Übereinkünften und Verträgen sowie damit in Zusammenhang stehende Organe und Institutionen die rechtliche Basis, auf Grund derer die Richtlinien und Prinzipien dieses Handbuchs zur Anwendung kommen können. Eine kurze Übersicht hierüber, angefangen bei den Genfer Konventionen, über die verschiedenen Organe der Vereinten Nationen, bis hin $\mathrm{zu}$ regionalen Abkommen und Organen auf inter-amerikanischer, europäischer und afrikanischer Ebene findet sich im ersten Kapitel, das sich mit den relevanten internationalen rechtlichen Standards befaßt.

Neben den rechtlichen Vorgaben spielen natürlich für alle diejenigen, die beruflich mit Folteropfern zu tun haben - also insbesondere wiederum Juristen und Juristinnen sowie Angehörige der Gesundheitsberufe, vor allem Ärzte und Ärztinnen -, auch die ethischen Kodizes der jeweiligen Berufsverbände eine herausragende Rolle. Diese werden in der gebotenen Kürze im zweiten Kapitel des Handbuchs behandelt. Außer den einschlägigen Bestimmungen der Berufsverbände, im Besonderen des Weltärztebundes (WMA), werden auch diejenigen Prinzipien näher ausgeführt, die weitgehend kultur- und zeitübergreifend allen Kodifizierungen der ethischen Verpflichtungen für Angehörige der Gesundheitsberufe gemeinsam sind: die Pflicht, anteilnehmende Fürsorge zu leisten, grundsätzlich eine Einwilligung des Patienten nach erfolgter Aufklärung (Informed consent) einzuholen und die ärztliche Schweigepflicht einzuhalten. Besonderes Augenmerk wird dem Problem der Doppelverpflichtung (Dual obligation/Dual loyalty) von medizinischem Fachpersonal gewidmet, das entweder bei der Polizei, im Gefängnis- oder Militärwesen arbeitet. 


\footnotetext{
I. RELEVANTE INTERNATIONALE RECHTLICHE STANDARDS

A. Humanitäres Völkerrecht

B. Die Vereinten Nationen

C. Regionale Organisationen

D. Der Internationale Strafgerichtshof

II. RELEVANTE ETHISCHE KODIZES

A. Berufsethik der Juristen

B. Ethik der Gesundheitsberufe

C. Grundsätze, die allen ethischen Kodizes der Gesundheitsberufe gemeinsam sind

D. Angehörige der Gesundheitsberufe mit Doppelverpflichtungen (,dual obligations')

III. RECHTLICHE UNTERSUCHUNG VON FOLTER

A. Ziele einer Untersuchung von Folter

B. Grundsätze für die wirksame Untersuchung und Dokumentation von Folter und anderer grausamer, unmenschlicher oder erniedrigender Behandlung oder Strafe

C. Verfahren einer Folteruntersuchung

D. Die Untersuchungskommission
}

\section{ALLGEMEINE HINWEISE FÜR INTERVIEWS}

A. Ziele der Ermittlung, Untersuchung und Dokumentation

B. Verfahrensorientierte Schutzmaßnahmen im Hinblick auf Häftlinge

C. Offizielle Besuche in Haftzentren

D. Fragetechniken

E. Dokumentation des Hintergrunds

F. Bewertung des Hintergrunds

G. Abklärung von Foltermethoden

H. Risiko einer Retraumatisierung der interviewten Person

I. Einsatz von Dolmetschern

J. Gender-Fragen

K. Indikationen für eine ärztliche Überweisung

L. Interpretation von Befunden und Schlußfolgerungen

V. PHYSISCHE BEWEISE FÜR FOLTER

A. Interviewstruktur

B. Krankengeschichte

C. Physische Untersuchung

D. Untersuchung und Beurteilung nach spezifischen Folterarten

E. Spezielle diagnostische Tests

VI. PSYCHOLOGISCHE NACHWEISE FÜR FOLTER

A. Allgemeine Hinweise

B. Psychische Folgen von Folter

C. Die psychologisch-psychiatrische Beurteilung

\section{ANHANG}

Übersicht: Struktur und Gliederung des Istanbul-Protokolls 
An dieser Stelle wird betont, daß alle Angehörigen der Gesundheitsberufe grundsätzlich den internationalen rechtlichen Vorgaben und ihrer Berufsethik verpflichtet bleiben, auch wenn ihr Arbeitgeber oder eine offizielle staatliche Stelle Handlungen fordert, die gegen die Rechte und das Wohl der Patienten gerichtet sind.

Das dritte Kapitel des Handbuchs thematisiert Verfahrensweisen und nennt Richtlinien für eine (straf)rechtliche Ermittlung bei einem Verdacht auf oder einer Anschuldigung von Folter. Besonders erwähnenswert sind hier die „Prinzipien einer wirksamen Untersuchung und Dokumentation von Folter und anderer grausamer, unmenschlicher oder erniedrigender Behandlung oder Strafe", die eigens noch einmal im ersten Anhang des Protokolls wiederholt werden: Sie stellen die Essenz und absoluten Minimalanforderungen dar, die jede ernsthafte Untersuchung eines Folterverdachts erfüllen muß. Daneben werden zahlreiche Hinweise zur Ausführung eines Ermittlungsverfahrens gegeben, die von der Bestimmung eines geeigneten Ermittlungsorgans, über die Befragung von Zeugen und die Sicherung von Beweisen bis hin zum Einsatz der Fotografie reichen. Abgeschlossen wird das Kapitel durch umfangreiche Erläuterungen, welche die $\mathrm{Zu}$ sammensetzung und Arbeit einer unabhängigen Untersuchungskommission regeln.

Nicht nur an Ärzte und Ärztinnen sowie Juristen und Juristinnen, sondern an alle diejenigen, die mit der Untersuchung von Menschenrechtsverletzungen befaßt sind, wendet sich das vierte Kapitel, das allgemeine Hinweise für die Befragung von Folteropfern gibt. Wichtig sind hier zum einen speziell die Hinweise zur Befragung von noch inhaftierten Personen, da diese in besonderer Weise gefährdet sind, im Verlauf einer Untersuchung (weitere) Repressalien befürchten zu müssen, sowie zum anderen die Überlegungen bezüglich des Risikos einer Retraumatisierung von Folteropfern durch die Situation eines möglicherweise schwierige Erinnerungen weckenden Gesprächs. Darüber hinaus finden sich in dem Kapitel unter anderem Überlegungen zu allgemeinen Fragetechniken, zu Problemen bei dem Einsatz von Dolmetschern und zu Gender-Fragen sowie eine Liste mit gebräuchlichen Foltermethoden.

Die beiden letzten Kapitel behandeln die Möglichkeiten eines körperlichen und psychologischen Nachweises von Folter und richten sich damit speziell an Ärzte und Ärztinnen sowie Psychologen und Psychologinnen.

In Kapitel fünf wird ausführlich die körperliche Untersuchung eines Folteropfers behandelt und die zu erwartenden Symptome sowohl nach betroffenen Körperregionen als auch entsprechend spezifischer Arten der Folter aufgeschlüsselt. Außerdem werden Diagnosemittel sowie eine mögliche Differenzialdiagnostik benannt.

Das sechste Kapitel über die Beurteilung psychologischer Beweise für Folter ist das ausführlichste des gesamten Handbuchs. Darin wird zunächst einmal die zentrale Rolle einer psychologischen Begutachtung von Folteropfern hervorgehoben, bevor die möglichen psychischen Folgen von Folter genannt und beschrieben werden. Anschließend folgen umfangreiche Hinweise zur Durchführung von psychologischen oder psychiatrischen Begutachtungen, in denen noch einmal spezifisch auf die Probleme des Interview-Prozesses eingegangen wird sowie auch auf die Möglichkeit von Übertragungs- und Gegenübertragungseffekten. Die notwendigen Komponenten einer psychologischen Untersuchung werden erläutert und der Einsatz von neuropsychologischen Testverfahren diskutiert. Abgeschlossen wird dieser Teil durch einige Bemerkungen zu den psychischen Auswirkungen von Folter auf Kinder.

Speziell an Ärzte und Ärztinnen wenden sich auch die Anhänge II bis IV. Der zweite Anhang diskutiert noch einmal die Anwendung verschiedener radiologischer Diagnoseverfahren sowie die Möglichkeit der Biopsie bei einer Folter durch Strom. Der dritte Anhang liefert eine Reihe von Skizzen des menschlichen Körpers, die für die Dokumentation von Folter und anderer 
Arten der Mißhandlung verwendet werden können. Der vierte Anhang schließlich stellt eine Vorlage dar, die als Formblatt für die medizinische Untersuchung benutzt werden kann und gibt den untersuchenden Ärzten und Ärztinnen damit noch einmal eine Richtlinie für die Dokumentation des Ablaufs einer solchen Untersuchung an die Hand.

Daß das Istanbul-Protokoll zwar in unterschiedlichen Kontexten Verwendung finden kann, sich in erster Linie aber doch an Juristen und Juristinnen sowie Ärzte und Ärztinnen richtet, wird aus der Einteilung der Kapitel klar. Von den sechs Kapiteln und vier Anhängen, aus denen das Handbuch besteht, wenden sich die zwei umfangreichsten Kapitel (zur körperlichen und psychologischen Untersuchung von Folteropfern) sowie drei Anhänge an Mediziner. Zwei Kapitel - zur rechtlichen Ermittlung bei Folter und im Prinzip auch das Kapitel über die relevanten internationalen rechtlichen Standards - sowie der erste Anhang, der noch einmal die wichtigsten Prinzipien einer (straf-) rechtlichen Ermittlung wiederholt, wenden sich an Juristen und Juristinnen. Das Kapitel über ethische Kodizes behandelt sowohl die Berufsethik von Juristen und Juristinnen als auch von Angehörigen der Gesundheitsberufe, so daß vor allem das Kapitel mit den allgemeinen Hinweisen für eine Befragung von Folteropfern (und in gewissem Maß auch das Kapitel über internationale Rechtsstandards) einen breiteren Kreis von Menschenrechtlern und Menschenrechtlerinnen anspricht. Die herausragende Bedeutung von Ärzten und Ärztinnen und Juristen und Juristinnen bei der Untersuchung von Foltervorfällen sowie die Notwendigkeit einer engen Zusammenarbeit dieser beiden Berufsgruppen spiegeln sich auch in den Bemühungen um eine Implementierung der durch das IstanbulProtokoll vorgegebenen Richtlinien und Standards.

\section{Umsetzung und Implementierung}

Gleich nach seiner Veröffentlichung gab es vor allem in der Türkei zahlreiche Bemühungen, die Richtlinien und standardisierten Verfahrensweisen des IstanbulProtokolls einem größeren Kreis medizinischen und juristischen Fachpersonals bekannt zu machen. Durch gemeinsame Ausbildungseinheiten von Ärzten und Ärztinnen und Rechtsexperten und Rechtsexpertinnen sollte sowohl das Wissen um das Protokoll und seine Anwendung in der täglichen Praxis verankert, als auch eine Motivation geschaffen werden, die Haltung gegenüber der Folter zu verändern. Letzteres zielte darauf $a b$, daß Hinweise auf Folter nicht mehr länger ignoriert, sondern vielmehr unter Berücksichtigung der Prinzipien des Istanbul-Protokolls weiterverfolgt würden. Die Trainingsseminare wurden gemeinsam von der TIHV, der Türkischen Ärztekammer (Turkish Medical Association/TMA) und der Gesellschaft gerichtsmedizinischer Experten (Society of Forensic Medicine Specialists/SFMS) ausgeführt, dem türkischen Team, das schon an der Entstehung des Protokolls beteiligt war. ${ }^{25}$

In den Jahren 2003 bis 2005 fand außerdem die erste Phase des "Istanbul Protocol Implementation Projects" (IPIP) statt. Dieses von der Europäischen Kommission geförderte Projekt wurde von dem International Rehabilitation Council for Torture Victims (IRCT) und dem Weltärztebund (World Medical Association/WMA) ins Leben gerufen. Ziel des Projektes war es, in fünf Pilotländern mit der Hilfe weiterer internationaler und lokaler Partner Mitarbeitende des Gesundheitswesens und Juristen und Juristinnen in der Anwendung des Istanbul-Protokolls $\mathrm{zu}$ schulen, um auf diese Weise sukzessive einen Rahmen für die allgemeine Einführung des Protokolls zu schaffen. Außerdem wurde eine Reihe von Materialien für die länder- und berufsspezifische Umsetzung des Protokolls erarbeitet. Während der ersten Phase des Projekts 
besuchten in Sri Lanka, Georgien, Uganda, Marokko und Mexiko insgesamt 244 Angehörige des Gesundheitswesens und 123 Anwälte, Anwältinnen und Justizangestellte die Trainingsseminare. Eine zweite Phase des Projekts wird ebenfalls von der EU finanziert. Fortgeführt wurde das IPIP zwischen 2005 und 2007 auch durch das "Prevention Through Documentation Project", das als weiteres Ziel die noch stärkere Förderung der Kooperation von Medizinern und Juristen und Juristinnen bei der Umsetzung des Istanbul-Protokolls sowie die Bereitstellung von Wissen über eine wirksame Prävention von Folter in den Rehabilitationszentren für Folteropfer hatte. Außer den bereits genannten Ländern waren hierbei noch Ägypten, Ecuador, Kenia, die Philippinen und Serbien beteiligt. Während in den neu hinzugekommenen Ländern wiederum Juristen und Juristinnen und Ärzte und Ärztinnen in der Anwendung des Protokolls geschult werden sollten, wurden in den bereits im IPIP beteiligten Ländern auch Trainer und Multiplikatoren ausgebildet. 26

Neben dem Einsatz des Istanbul-Protokolls in Ländern, in denen Folter weiterhin systematisch ausgeübt wird oder zumindest weit verbreitet ist, bietet es jedoch auch in anderen Staaten eine wirksame Hilfestellung für Menschenrechtsorganisationen und psychosoziale Zentren bei ihrer Arbeit mit Folterüberlebenden. Darüber hinaus können die Richtlinien des Protokolls in Asylverfahren verwendet werden, wenn eine Begutachtung von Flüchtlingen und eventuell der Nachweis einer mutmaßlichen, unter Umständen bereits länger zurückliegenden Folter erbracht werden soll.

Diesem Ziel entsprechend haben die Physicians for Human Rights (PHR) in den Vereinigten Staaten von Amerika auf der Grundlage des Istanbul-Protokolls 2001 ein eigenes Handbuch zur Untersuchung von

26 Ebd., S. 266f. Weitere Informationen sind auch auf der Internet-Seite des International Rehabilitation Councils for Torture Victims verfügbar: www.irct.org (22. September 2008).
Asylbewerbern erstellt. ${ }^{27}$ Dabei wurden die Teile des Istanbul-Protokolls, die für eine Begutachtung mutmaßlich gefolterter Menschen im Zusammenhang mit Asylverfahren relevant sind, beibehalten und um zwei Teile ergänzt, von denen der eine sich spezifisch mit der Asylrechtssituation in den USA befaßt, während der andere eine Reihe beispielhafter eidesstattlicher Gutachten anführt.

Auch das niederländische Projekt "CARE FULL" beschäftigt sich mit der Anwendung des Protokolls in Asylverfahren. Es wurde initiiert von Amnesty International Niederlande, dem Dutch Council for Refugees und Pharos (Knowledge Centre on Refugees and Health) und entstand aus der Sorge, daß die Opfer von Folter und Mißhandlungen aufgrund der in der gesamten EU immer strikter werdenden Asylrechtspraxis kein entsprechendes Gehör mehr finden. ${ }^{28}$ Innerhalb des Projekts wurde untersucht, inwieweit das Istanbul-Protokoll schon Anwendung in Asylverfahren innerhalb der EU findet und wie es in solchen Verfahren einzusetzen ist. Die klare Schlußfolgerung aus der Untersuchung: Medizinisch-psychologische Gutachten, die eine Folterbehauptung zumindest weitgehend entweder bestätigen oder auch widerlegen können, müssen in Asylverfahren ein angemessenes Gewicht erhalten. Außerdem sollten diese Gutachten nach den im Istanbul-Protokoll aufgestellten Kriterien durchgeführt werden. ${ }^{29}$

27 Physicians for Human Rights, Examining Asylum Seekers. A Health Professional's Guide to Medical and Psychological Evaluations of Torture, 2001. Online erhältlich unter: http:// physiciansforhumanrights.org/library/ documents/reports/examining-asylumseekers-a.pdf (22. September 2008).

28 Bruin/Reneman/Bloemen (Fn. 18).

29 Siehe hierzu auch eine Broschüre mit den aus dem CARE FULL-Projekt gewonnen Prinzipien und Empfehlungen: René Bruin/Marcel Reneman/Evert Bloemen, CARE FULL. Medico-legal reports and the Istanbul Protocol in asylum procedures: Principles and Recommendations, Amsterdam/Utrecht 2006. Online erhältlich unter: $\quad$ www.pharos.nl/uploads/_site_1/Pdf/ 
Das dänische "Rehabilitation and Research Center for Torture Victims" hat in einem Pilotprojekt untersucht, inwiefern sich eine medizinisch-rechtliche Dokumentation nach den Standards des Istanbul-Protokolls im Rahmen der therapeutischen Behandlung von Folteropfern durchführen läßt. Als Ergebnis konnte gezeigt werden, daß es erhebliche Synergieeffekte gibt, da ein Großteil der für einen rechtlichen Zweck notwendigen Daten bereits während des Rehabilitationsprozesses gewonnen wird. Allerdings besteht dennoch ein gewisser Mehraufwand, da sowohl noch zusätzliche Daten erhoben als auch die bereits gewonnen Daten restrukturiert und zugänglich gemacht werden müssen, wodurch die Erarbeitung eines eigenen Konzepts für die Speicherung der Daten notwendig wird. Weil der Nutzen einer medizinischrechtlichen Dokumentation von Folter allerdings wissenschaftlich bisher wenig erforscht ist, bleibt die Frage offen, ob der zu erwartende Mehraufwand gerechtfertigt ist. 30

\section{Schlußüberlegungen}

Trotz der Auszeichnung durch die Vereinten Nationen, verschiedener internationaler Organe $^{31}$ und der vielfältigen Aktivitäten zur Verbreitung des Wissens um das Pro-

Documenten/Care\%20Full\%20PenR.pdf September 2008).

30 Siehe hierzu Mandel/Worm (Fn. 8) und zu den Ergebnissen des Pilotprojekts insbesondere dies., Documentation of torture victims, assessment of the Start Procedure for Medico-Legal Documentation, in: Torture 2007, Vol. 17/3, S. 196-202.

31 Beispielsweise hat der Sonderberichterstatter über Folter in seinen allgemeinen Empfehlungen noch einmal auf die Wichtigkeit der in dem Istanbul-Protokoll aufgestellten Prinzipien hingewiesen (E/CN.4/2003/68, § 26) und die Menschenrechtskommission hat in ihrer Resolution zu den Menschenrechten und der Gerichtsmedizin die Staaten aufgefordert, das Handbuch als ein nützliches Werkzeug im Kampf gegen Folter einzusetzen (Resolution 2003/33 vom 23. April 2003; E/CN.4/2003/ L.11/Add.4). Siehe hierzu auch den Beitrag von Ucpinar/Baykal (Fn. 21), S. $256 f$. tokoll, 32 hat das Handbuch bis heute nicht den ihm zustehenden Stellenwert erlangt. ${ }^{33}$ Auch in Deutschland, Österreich und der Schweiz ist es bisher unter Ärzten und Ärztinnen und Rechtsexperten und Rechtsexpertinnen zu wenig bekannt. Da es außerdem keine einheitlichen Vorschriften darüber gibt, welche Qualifikationen eine Ärztin oder ein Arzt besitzen muß, um ein medizinisch-psychologisches Gutachten - falls überhaupt eines angefordert wird - in einem ausländerrechtlichen Verfahren erstellen zu können, finden das Protokoll und die in ihm enthaltenen Richtlinien in Deutschland noch kaum Anwendung. Abhilfe in bezug auf einheitliche Richtlinien für die Begutachtung im Rahmen von Asylverfahren schaffen seit einiger Zeit die in der Arbeitsgruppe um Dr. Gierlichs (Aachen) und Dr. Wirtgen (München) entstandenen "Standards zur Begutachtung psychisch reaktiver Traumafolgen. “34 Diese Empfehlungen legen die fachlichen Voraussetzungen fest, die ein Arzt, der als Gutachter in einem Asylverfahren tätig sein will, besitzen muß, um seinen Auftrag sorgfältig und ordnungsgemäß ausführen zu können. In Zusammenarbeit mit den Ärztekammern und den Kammern für Psychologen werden außerdem nach einem festen Fortbildungscurriculum bundesweit zertifizierte Fortbildungsveranstaltungen durchgeführt. Entstanden ist außerdem ein

32 Im September 2003 hat der Weltärztebund eine Erklärung zur „Verantwortung von Ärzten bei der Verurteilung von Folterakten oder grausamer, unmenschlicher oder erniedrigender Behandlungen, die ihnen zur Kenntnis gelangt sind" verabschiedet. Darin empfiehlt er in Absatz 20 den nationalen Ärzteorganisationen, „ihren Ärzten das Istanbul-Protokoll zur Verfügung“ zu stellen. Weltärztebund, Handbuch der Deklarationen, Erklärungen und Entschließungen, 2004, S. 251. Erhältlich unter www.bundesaerztekammer.de/downloads/ha ndbuchwma.pdf (22. September 2008).

$33 \mathrm{Zu}$ den möglichen Gründen hierfür siehe: Mandel/Worm (Fn. 8), S. 18f.

34 Die Projektgruppe nennt sich „Standards zur Begutachtung psychotraumatisierter Menschen" (SBPM). Weitere Informationen sind auf der Internet-Seite der SBPM verfügbar: www.sbpm.de/ (22. September 2008). 
eigenes Handbuch für die psychologische Begutachtung von Asylbewerbern, das von Dr. Haenel und Dr. Wenk-Ansohn herausgegeben wurde. ${ }^{35}$

Dennoch kann der Einsatz des IstanbulProtokolls, vor allem, wenn er komplementär verstanden wird, durchaus sinnvoll sein, da es noch über diese Standards hinausgeht. Beispielsweise finden sich sehr detaillierte Hinweise zum Einsatz von Dolmetschern und neben einem Kapitel über die psychologische Untersuchung mutmaßlicher Folteropfer enthält es, wie bereits geschildert, auch eine differenzierte Beschreibung möglicher körperlicher Symptome spezifischer Folterarten, der anzuwendenden Diagnosemittel sowie einer möglichen Differenzialdiagnostik. Natürlich finden sich in dem vorliegenden Handbuch auch Richtlinien und Teile, die im westeuropäischen Kontext und im Rahmen von Asylverfahren nicht erforderlich sind. Genannt seien hier nur die Ausführungen $\mathrm{zu}$ den internationalen rechtlichen Regelungen des Folterverbotes und die Beschreibung von Verfahren für eine juristische Ermittlung gegen die Täter. Da das Protokoll im deutschsprachigen und europäischen Raum seine Anwendung derzeit vorrangig in Asylverfahren finden wird, wo es um die bloße Feststellung eines Asylgrundes, nicht um die Strafverfolgung möglicher Täter geht, sind die entsprechenden Ausführungen hier aktuell von eher untergeordneter Bedeutung. ${ }^{36}$ Aus

35 Ferdinand Haenel/Mechthild Wenk-Ansohn (Hrsg.), Begutachtung psychisch reaktiver Traumafolgen in aufenthaltsrechtlichen Verfahren, 2005. Siehe bereits Angelika Birck, Wie krank muß ein Flüchtling sein, um von der Abschiebung ausgenommen $\mathrm{zu}$ werden, in: MRM 2000, S. 106118.

36 In einem Papier, das die beiden bereits zitierten Aufsätze zusammenführt, haben Lene Mandel und Lise Worm noch einmal die Situationen für eine mögliche Verwendung einer medizinischrechtlichen Dokumentation von Foltervorfällen entsprechend der Standards des IstanbulProtokoll auch innerhalb von Staaten, in denen selbst nicht gefoltert wird, dargestellt. Dies können neben Asylverfahren sein: Um gegen die Straffreiheit von Tätern zu kämpfen (durch internationale oder auch nationale Strafverfah- diesem Grund war zunächst eine Überlegung, in Anlehnung an das von den PHR herausgegebene Buch "Examining Asylum Seekers", auch in der deutschen Übersetzung des Istanbul-Protokoll eventuell nur die für Asylverfahren relevanten Teile herauszugeben und um Beiträge zu ergänzen, die sich mit der konkreten Asylrechtspraxis in Deutschland, Österreich und der Schweiz befassen. Letztendlich erscheint es aber sinnvoller, das Protokoll zumindest einmal in seiner Gesamtheit vorzulegen, um es unabhängig von einem spezifischen Verwendungszweck im deutschsprachigen Raum bekannt zu machen und so hoffentlich einer breiteren Verwendung zuzuführen. In jedem Fall wird es sehr gut möglich sein, sich bei Bedarf die benötigten Richtlinien und Prinzipien, beispielsweise zur psychologischen Begutachtung eines mutmaßlichen Folteropfers, aus dem Text zusammenzustellen. Insgesamt soll die deutsche Übersetzung des Istanbul-Protokolls dazu beitragen, das Bewußtsein vor allem unter Ärzten und Ärztinnen und Juristen und Juristinnen für die Probleme von Folteropfern zu schärfen. Eine Konfrontation mit diesen Problemen kann nämlich nicht nur während eines möglichen Asylverfahrens oder einer damit zusammenhängenden Begutachtung auftreten, sondern ebenso im Rahmen einer "normalen“ medizinischen Behandlung eines Folterüberlebenden, der entweder noch auf den Ausgang seines Asylverfahrens wartet oder dessen Antrag gegebenenfalls bereits genehmigt wurde.

\section{Anhang}

Folgende Organisationen waren bei der Entwicklung des Istanbul-Protokolls beteiligt:

ren); um Forschung über die Folgen von Folter und über möglichen Strategien gegen Folter durchzuführen; um Lobby-Aktivitäten zu untermauern; um Fürsprache-Aktivitäten für Folteropfer zu untermauern; und um Dokumentationsmethoden weiter $\mathrm{zu}$ entwickeln. Siehe: Lene Mandel/Lise Worm, Implementing the Istanbul Protocol, Praxis Paper No. 3, Kopenhagen 2006, S. 6 und S. 11ff. Ebenso dies., (Fn. 8, Fn. 30). 
Action for Torture Survivors (HRFT), Genf, Amnesty International, London; Association for the Prevention of Torture, Genf; Behandlungszentrum für Folteropfer, Berlin; British Medical Association (BMA), London; Center for Research and Application of Philosophy and Human Rights, Hacettepe University, Ankara, Center for the Study of Society and Medicine, Columbia University, New York, Centre Georges Devereux, Université de Paris VIII, Paris, Committee against Torture, Genf, Danish Medical Association, Kopenhagen, Department of Forensic Medicine and Toxicology, University of Colombo, Colombo, Ethics Department, Dokuz Eylül Medical Faculty, Izmir, TürkeI, Gaza Community Mental Health Programme, Gaza, Bundesärztekammer, Berlin, Human Rights Foundation of Turkey (HRFT), Ankara, Human Rights Watch, New York, Indian Medical Association and the IRCT, Neu Delhi, Indochinese Psychiatric Clinic, Boston, USA, Institute for Global Studies, University of Minnesota, Minneapolis, USA, Instituto Latinoameri- cano de Salud Mental, Santiago, International Committee of the Red Cross, Genf, International Federation of Health and Human Rights Organizations, Amsterdam, Niederlande, International Rehabilitation Council for Torture Victims (IRCT), Kopenhagen, Johannes Wier Foundation, Amsterdam, Niederlande, Lawyers Committee for Human Rights, New York, Physicians for Human Rights Israel, Tel Aviv, Physicians for Human Rights Palestine, Gaza, Physicians for Human Rights USA, Boston, Program for the Prevention of Torture, InterAmerican Institute of Human Rights, San José, Society of Forensic Medicine Specialists, Istanbul, Türkei, Special Rapporteur on Torture, Genf, Survivors International, San Francisco, USA, The Center for Victims of Torture (CVT), Minneapolis, USA, The Medical Foundation for the Care of Victims of Torture, London, The Trauma Centre for Survivors of Violence and Torture, Cape Town, Südafrika, Turkish Medical Association, Ankara, World Medical Association, Ferney-Voltaire, Frankreich. 